\title{
El feminismo de la gobernanza en la CEDAW: la cuestión sobre el trabajo sexual y la prostitución*
}

\author{
Felipe JARAMILLO RUIZ** \\ Lina-María CÉSPEDES-BÁEZ****
}

\section{Resumen}

A partir del debate sobre el feminismo de la gobernanza, este artículo investiga las recomendaciones generales del Comité de la Convención sobre la Eliminación de Todas las Formas de Discriminación contra la Mujer (CEDAW) y las observaciones finales de los informes de los Estados parte presentadas ante el mismo. El objetivo consiste en analizar el enfoque que este órgano ha incorporado para abordar la comercialización del sexo desde una perspectiva crítica. En este sentido, se encuentra que el Comité de la CEDAW ha promovido un enfoque de descriminalización parcial o abolicionista que pretende penalizar a aquellos que participen en el comercio sexual, salvo a la mujer en prostitución, la cual es considerada primordialmente como una víctima. Así, el artículo provee hallazgos empíricos que permiten entender las aproximaciones a la sexualidad que predominan en la regulación $y$ organismos internacionales $y$ sus posibles implicaciones para el feminismo de la gobernanza.

Palabras clave: Feminismo de la Gobernanza, Prostitución, Trabajo Sexual, CEDAW, Derechos Humanos..

* Recebido em 10 de novembro de 2019, aceito em 20 de maio de 2020.

** Ph.D. Affiliate of the Gender Center, Graduate Institute of Geneva, Ginebra, Suiza. felipe.jaramillo@graduateinstitute.ch / https://orcid.org/0000-0003-3766$\underline{3484}$

*** Profesora, Facultad de Jurisprudencia, Universidad del Rosario, Bogotá, Colombia. linam.cespedes@urosario.edu.co / https://orcid.org/0000-0002-56020559 
Governance Feminism at CEDAW: the Question of Sex Work and Prostitution

\begin{abstract}
Based on a discussion of governance feminism, this article analyzes the general recommendations and concluding observations of the United Nations Committee on the Elimination of Discrimination against Women (CEDAW). The objective is to critically analyze the approach that this body has used to address the commercialization of sex. In this sense, we find that the CEDAW Committee has promoted an abolitionist approach or one favoring partial decriminalization, which penalizes those who participate in the sex trade, except women in prostitution, who are primarily seen as victims. Thus, the empirical findings serve to understand the approaches to sexuality that prevail in international norms and organizations and their possible implications for governance feminism.
\end{abstract}

Keywords: Governance Feminism, Prostitution, Sex Work, CEDAW, Human Rights. 


\section{Introducción}

A partir del concepto de "feminismo de la gobernanza," el cual da cuenta de las formas en que las ideas feministas logran ejercer poder a través de diversas estructuras y proyectos sociales (Halley et al. 2018, ix), este artículo examina cómo el Comité de la Convención sobre la Eliminación de Todas las Formas de Discriminación contra la Mujer (de ahora en adelante Comité de la CEDAW, por sus siglas en inglés) aborda la comercialización del sexo. Nuestro análisis está enfocado únicamente en el fenómeno de la comercialización del sexo entre adultos ${ }^{1}$ y en la manera cómo el Comité de la CEDAW lo ha abordado en cumplimiento de su función de interpretar la Convención sobre la Eliminación de todas las Formas de Discriminación contra la Mujer. Al explorar de forma sistemática la aproximación a la comercialización del sexo por parte de este órgano, se provee insumos empíricos que permiten comprender las características del feminismo de la gobernanza. Así, la investigación aporta a la discusión sobre qué tipo de ideas feministas logran incursionar en las estructuras de poder internacional y revela los principales enfoques que se incorporan frente a la comercialización del sexo.

El término feminismo de la gobernanza fue acuñado en 2006 por Janet Halley et al. (2006) para reflexionar sobre la inserción de las ideas feministas en las estructuras de poder internacional. Para las autoras, a medida que las ideas feministas se integran a la gobernanza global, el feminismo se sumerge en relaciones de poder y participa en la formulación de tecnologías legales. Así, los proyectos feministas pasan de ocupar una posición al margen y de resistencia a intervenir en la creación de regímenes normativos internacionales. A partir del concepto del feminismo de la gobernanza, las autoras invitan a identificar qué clase de ideas y líneas de acción feministas se incrustan en el poder, para luego

\footnotetext{
${ }^{1}$ La Convención sobre los Derechos del Niño de Naciones Unidas define en su artículo $1^{\circ}$ como "niño todo ser humano menos de dieciocho años de edad (...)".
} 
cadernos pagu (59), 2020:e205917 El feminismo de la gobernanza en la CEDAW

ponderar sus aciertos, efectos inesperados y alteraciones en la distribución del poder.

Desde que Janet Halley et al. (2006) acuñaron el concepto del feminismo de la gobernanza, diversas autoras han examinado la incorporación de las ideas feministas en estructuras de poder. Dianne Otto (2010a, 2010b) analiza el alcance de las ideas feministas en las resoluciones del Consejo de Seguridad de la Organización de Naciones Unidas. Hilary Charlesworth (2011) evidencia los límites legales y políticos de los avances feministas en materia de regulación de los conflictos armados. Elisabeth Prügl (2017, 2011) investiga la cooptación de las ideas feministas en las políticas económicas internacionales. Lina Céspedes-Báez (2014) y Sussanne Zwingel (2014) exploran cómo las agendas y demandas feministas logran incidir en los marcos normativos y políticas nacionales. Sin embargo, aunque la discusión en torno al feminismo de la gobernanza ha suscitado grandes debates en las principales revistas académicas anglosajonas, poco se ha escrito en español sobre el tema (Céspedes-Báez, 2014). Por consiguiente, el artículo evidencia la importancia de traer la discusión sobre el feminismo de la gobernanza a las agendas investigativas de la academia hispanohablante y se materializa como un avance en el debate sobre las narrativas transnacionales del movimiento feminista.

En términos metodológicos, se examinaron las 37 recomendaciones generales que se han adoptado desde 1986, año en que el Comité de la CEDAW publicó la primera recomendación general, hasta el 13 marzo de 2018, fecha de la última recomendación general al momento de redacción de este artículo. Además, se analizaron las observaciones finales sobre los informes periódicos de los Estados parte del Comité desde enero de 2000 hasta marzo de 2018. En total, se estudiaron 425 observaciones finales de más de 175 Estados. Por medio del software de análisis cualitativo NVivo, se realizó una consulta por medio de dos palabras clave, con el fin de realizar la búsqueda y codificación: prostitución y trabajo sexual. Esta decisión metodológica generó ciertos puntos ciegos, en tanto que el fenómeno de la 
comercialización del sexo no se reduce a la prostitución y al trabajo sexual. Es decir, existen otras cuestiones que también están relacionadas con la comercialización del sexo, como lo son el turismo sexual, el tráfico sexual, la trata de personas y la explotación sexual. Así, al enfocarse en el uso de los términos prostitución y trabajo sexual, el estudio sólo discute estos fenómenos si se mencionan en los extractos que hacen referencia a las dos palabras clave. Como toda investigación de carácter inductivo, los principales argumentos surgieron a partir de un proceso interpretativo y se desarrollaron conforme a las temáticas que se identificaron en los textos.

En estos términos, la investigación permitió identificar los documentos en los cuales el Comité de la CEDAW se refiere a la comercialización del sexo. Dichas referencias se codificaron de acuerdo con los enfoques propuestos por Halley et al. (2006). Es decir, se identificó si en estas referencias se propendía por la criminalización total, la abolición o descriminalización parcial, la descriminalización completa o la legalización de la comercialización del sexo. En suma, la investigación permitió rastrear la frecuencia de ciertas temáticas y respuestas frente a la comercialización del sexo. Al ser esta una investigación de carácter interpretativo, los argumentos centrales se construyeron a partir de una lectura crítica de los documentos codificados y la literatura feminista. En este sentido, los patrones que se identificaron están íntimamente relacionados al proceso de codificación.

El artículo está dividido en dos secciones. Primero, se explica el concepto del feminismo de la gobernanza y la polémica que este ha desencadenado entre las autoras feministas. Segundo, se exponen los resultados de la investigación. Así mismo, al contrastar los elementos conceptuales del feminismo de la gobernanza con los hallazgos, se analiza cómo las recomendaciones y observaciones del Comité de la CEDAW brindan una visión más compleja sobre la inserción de las ideas feministas en las instituciones internacionales de derechos humanos. 
cadernos pagu (59), 2020:e205917 El feminismo de la gobernanza en la CEDAW

\section{El feminismo de la gobernanza y la comercialización del sexo}

El término feminismo de la gobernanza captura la forma en que ciertas ideas feministas dejan de ser simples reivindicaciones elevadas frente a centros de poder, como el Estado, para lograr la eliminación de la discriminación y violencia en contra de las mujeres, y se convierten en parte de los mecanismos que regulan la conducta de los seres humanos (Halley et al. 2018). La utilidad de esta categoría radica en que no solo permite identificar cómo ocurre el tránsito que incorpora el feminismo dentro de las dinámicas de gobernanza, sino que habilita el análisis de sus consecuencias. Poner énfasis sobre los efectos de la incorporación de las ideas feministas en las tecnologías de la regulación de la conducta humana implica reconocer que el feminismo aspira a ejercer poder y que, al lograrlo, redefine las distribuciones del mismo (Halley et al., 2018).

En este sentido, la categoría feminismo de la gobernanza toma como punto de partida dos supuestos:

(i) Si bien todos los proyectos feministas persiguen erradicar la discriminación y violencia en contra de todo aquello considerado femenino, existe variación en las maneras de entender cómo se producen estas situaciones y de encontrar soluciones acordes. Las variaciones responden a los puntos de partida teóricos propios de cada vertiente feminista. Así, por ejemplo, el feminismo liberal más clásico aboga por lograr la igualdad formal a través de las reformas legales, por cuanto identifica como fuente primordial de discriminación y violencia de lo femenino las diferencias creadas a través de las normas jurídicas y sociales entre hombres y mujeres (MacKinnon, 1983). Por su parte, las feministas de la diferencia o culturales no buscan una eliminación de las diferencias en la regulación, sino la incorporación de estas, a través de una reflexión que permita entender que allí radica lo valioso de lo femenino (Dietz, 2003; 
Crenshaw, 1991). El llamado feminismo del tercer mundo promueve una aproximación que problematiza la noción de mujer unidimensional que promueven los feminismos blancos de élite estructurados en centros hegemónicos de pensamiento (Mohanty, 1988; Narayan, 2006). Igualmente, reta la imagen que estos han creado del sujeto del tercer mundo como despojado de agencia (Verschuur, 2014). De ahí que su comprensión de la discriminación y violencia contra las mujeres se relacione directamente con el colonialismo, la clase y la raza, y que su objetivo de erradicación de estos fenómenos pase por la reivindicación de las iniciativas locales que se construyen desde las bases.

(ii) El tener como objetivo erradicar la discriminación y la violencia en contra de las mujeres y todo aquello identificado como femenino implica subvertir la distribución de poder (MacKinnon, 1983). En este sentido, el feminismo aspira a regular de manera directa $y / 0$ indirecta la conducta de los seres humanos, ya sea desde el Estado, la comunidad o el sector privado, entre otros (Prügl, 2011). Aceptar que el feminismo ejerce poder conlleva a que se puedan apreciar las ventajas y desventajas de sus estrategias. El hecho de que el feminismo promueva la erradicación de situaciones de vejatorias de lo femenino no implica que todas sus acciones para conseguir sus objetivos están libres de controversia o de consecuencias inesperadas (Otto, 2010b). Esto permite acercarse a los proyectos feministas desde una perspectiva crítica y examinar de manera minuciosa los efectos esperados $e$ inesperados que se siguen de la implementación de sus propuestas (Halley et al., 2018).

En este marco de ideas, el feminismo de la gobernanza permite rastrear qué ideas feministas logran convertirse en dispositivos efectivos de regulación de la vida social y determinar 
cadernos pagu (59), 2020:e205917 El feminismo de la gobernanza en la CEDAW

sus impactos (Halley, 2008). Así, esta categoría contribuye al entendimiento de cómo los proyectos feministas viajan, traspasan fronteras, son incorporados en el derecho y se consolidan en conocimientos especializados, y cuáles son las transformaciones que sufren cuando son adoptados y utilizados por individuos, comunidades, Estados u organizaciones (Halley et al., 2018). Por esto, este concepto implica un proyecto feminista crítico que pretende hacer visible cuál es la responsabilidad política que se sigue cuando la teoría se convierte en prácticas de gobernanza (Otto, 2010b; Charlesworth, 2008).

Uno de los primeros usos del feminismo de la gobernanza para el estudio de los impactos de las ideas feministas en la comprensión y la regulación de fenómenos relacionados con discriminación y violencia en contra de las mujeres se dio en el contexto de la comercialización del sexo. Con este propósito, Halley et al. (2006) identifican cuatro posibles aproximaciones a la regulación de este fenómeno, con el fin de determinar qué tipo de feminismo(s) había(n) logrado influenciar la producción de regulación jurídica a nivel internacional y local ${ }^{2}$ :

1. La "criminalización total" de todo tipo de trabajo sexual, por medio de la cual se sanciona penalmente la venta y compra de sexo y cualquier tipo de participación por parte de terceros (el proxeneta, burdel y consumidor).

2. La "abolicionista o descriminalización parcial", la cual no penaliza a las trabajadoras sexuales, pero sanciona penalmente a todos los demás actores involucrados en la industria sexual, incluyendo a los consumidores. Tal como Halley et al. (2006) anotan, las mujeres que ejercen la prostitución no son sometidas a las consecuencias del derecho

\footnotetext{
${ }^{2}$ A continuación, presentamos las cuatro aproximaciones propuestas por Halley et. al (2006), para lo cual parafraseamos el contenido específico de su caracterización. Para consultar el texto original en inglés ver las páginas 338-39 de ese artículo.
} 
penal en el entendido de que son víctimas de un sistema patriarcal.

3. La "descriminalización completa", por medio de la cual no se expiden normas criminales particulares para penalizar esta actividad, aunque ciertas conductas relacionadas con la misma pueden ser investigadas y castigadas por otras normas penales generales.

4. La "legalización", la cual consiste en la descriminalización total compaginada con provisiones legales que regulen las actividades relacionadas al trabajo sexual (Halley et al., 2006, 338-39).

En sus hallazgos, Halley et al. (2006) señalan que el proyecto de la criminalización completa parece no hacer parte de ninguna agenda propiamente feminista y que las discusiones a este nivel se han dado respecto de los tres restantes modelos. Así mismo, ponen en evidencia que la tendencia del feminismo de la gobernanza se ha inclinado a favor de una concepción de las mujeres como víctimas y de la criminalización de todos o algunos de los participantes en la comercialización del sexo como estrategia para abolirlo. De esta manera, las autoras demuestran que el feminismo que tiene el poder de la gobernanza exhibe un denominador común respecto de sus herramientas de intervención en esta área: la prohibición a través de la criminalización (Halley et al., 2018).

Para Halley et al. (2006), esto se explica por la influencia de la corriente del feminismo radical en la producción de regulación jurídica en torno a la comercialización del sexo. Las autoras argumentan que esta vertiente feminista ha logrado posicionar su explicación sobre los orígenes de la discriminación y violencia en contra de las mujeres y su estrategia para lograr su emancipación como la idea dominante en el feminismo de la gobernanza. En este sentido, sugieren que el feminismo de la gobernanza no ve el derecho penal como una última ratio sino que concibe la 
cadernos pagu (59), 2020:e205917 El feminismo de la gobernanza en la CEDAW

criminalización como un mecanismo fundamental para el avance de sus compromisos (Halley, 2016, 2008; Halley et al., 2018).

Este feminismo radical, al cual se refieren Halley et al. (2006), tiene sus orígenes en Estados Unidos de América en la década de 1980 y es fruto de una transformación del feminismo radical propio de finales de 1960 (Halley et al., 2018). Si bien estos dos feminismos radicales tienen puntos en común, como su llamado a una completa transformación social para ponerle fin a la discriminación y violencia contra las mujeres, y su identificación de la dominación masculina como el origen de todas las demás opresiones sociales, tales como las de clase o raza, el feminismo radical acuñado a partir de 1980 concibió la utilización del derecho y la consiguiente reforma legal como la herramienta y el camino para alcanzar sus fines (MacKinnon, 1983). Esto significó una transformación fundamental del pensamiento feminista radical de finales de 1960, en el que el Estado y su sistema jurídico eran objeto de suspicacia y objetivo de deconstrucción por cuanto eran otras manifestaciones de un régimen opresor (Dietz, 2003).

El feminismo radical nacido en 1980 es considerado como un feminismo estructuralista y legalista (Jaramillo Ruiz, 2013). Lo primero, por cuanto considera que la dominación masculina está presente y configura todas las relaciones sociales. Así, cualquier expresión de discriminación social puede y debe ser explicada solo en términos de lo masculino ejerciendo poder sobre lo femenino (Halley, 2008a). Lo segundo, en la medida en que concibe la reforma legal y el litigio como la estrategia indicada para subvertir esa situación (MacKinnon, 1983). Este feminismo explica que la subordinación de las mujeres se logra a través del control de su sexualidad (Jaramillo Ruiz y Erazo, 2016). La reforma jurídica a través del litigio y la actividad legislativa son elementos esenciales de la táctica para desmontar esta estructura de dominación (Halley et al., 2018). Así, los cuerpos jurídicos se han convertido en los vehículos privilegiados para promover cambios en la situación de las mujeres, y las abogadas en las figuras centrales capaces de transformar un pensamiento ajeno a las estructuras del poder en gobernanza (Otto, 2010a). 
Estas ideas básicas del feminismo radical han logrado tener eco en el derecho internacional, como en el caso de la producción de normas en materia de violencia sexual en el contexto de conflicto armado (Charlesworth, 2011; Monroy and Jaramillo Ruiz, 2017). Aunque era esencial hacer visible la gravedad de este delito a nivel de derecho penal internacional, la influencia de esta vertiente del feminismo es evidente en el posicionamiento de esta conducta delictiva como el crimen de género y en la reducción de la experiencia de las mujeres en estas situaciones a la victimización por crímenes relacionados con su sexualidad (Bindel, 2017; Lowenkron y Piscitelli, 2015).

Las propuestas feministas radicales han tenido amplia recepción en el derecho internacional, debido a que tienen vocación de universalidad y se acoplan fácilmente al discurso de los derechos humanos (Halley, 2016; Miller and Haltiwanger, 2004). Decir que la dominación masculina es una y estructural, permite ofrecer una explicación estándar para la situación de las mujeres sin importar en qué lugar del mundo se encuentren (Piscitelli, 2011). Su conceptualización del predicamento de las mujeres alrededor de una sexualidad subyugada e instrumentalizada por lo masculino permite mantener una noción conservadora de lo que es ser mujer y a la vez promover acciones para la protección de estos sujetos victimizados (Bernstein, 1999). Igualmente, el feminismo radical privilegia soluciones jurídicas que, si bien hacen visible la violencia que sufren las mujeres, no se relacionan necesariamente con las dimensiones económicas en que esta se inscribe (Piscitelli, 2014; Céspedes-Báez, 2014).

La preeminencia del feminismo radical en el escenario del derecho internacional no hubiera sido posible sin contar con una estructura de agencias dedicadas a la promoción y monitoreo de derechos humanos consolidada a nivel de Naciones Unidas, y un movimiento de organizaciones no gubernamentales con el conocimiento necesario para impactar esta institucionalidad (Halley et al., 2006; Monroy and Jaramillo Ruiz, 2017; Prügl, 2011). El reconocimiento de los derechos de las mujeres como derechos humanos, a finales de 1980 y principios de 1990, también permitió 
cadernos pagu (59), 2020:e205917 El feminismo de la gobernanza en la CEDAW

que este feminismo posicionará temas claves de su agenda en este ámbito (Zwingel, 2014). Debido a su énfasis en la dominación sexual masculina, el tráfico sexual y la prostitución fueron posicionados como problemáticas clave que una vez fueran resueltas minarían las bases estructurales de la jerarquía de género (Halley, 2016; Miller and Haltiwanger, 2004; Bernstein, 1999).

El feminismo radical ha considerado el comercio sexual como una de las expresiones más evidentes y representativas de la dominación masculina (Halley et al., 2006, 349; Kempadoo, 2005). En esta lectura no hay cabida para el consentimiento, pues las mujeres no pueden ejercer voluntariamente una actividad que es una de las máximas expresiones de la dominación sexual a la que están sometidas (Halley, 2016; MacKinnon, 1983; Piscitelli, 2011). En este sentido, la prostitución es entendida como una manifestación del tráfico sexual que debe ser abolida por medio de la criminalización de todos aquellos que la hacen posible y la protección de las mujeres que se han visto forzadas a ejercerla (Halley et al., 2006; Bindel, 2017; Vianna y Lowenkron, 2017).

La administración del presidente estadounidense George W. Bush fue determinante en darle preeminencia internacional a este enfoque por medio de la integración del tema en la política exterior de Estados Unidos (Stabile y Kumar, 2005; Choudhury, 2010). Así, inició el monitoreo de las medidas para combatir el tráfico sexual implementadas por los países receptores de sus fondos, y estableció como condición para las organizaciones que aplicaran a fondos de este país relacionados con VIH/SIDA y/o en contra del tráfico sexual el no promover la legalización de la prostitución (Bindel, 2017; Charlesworth, 2011; Halley et al., 2006).

En este momento, existen dos instrumentos jurídicos que han dado forma a la manera en que se entiende y trata el tema del tráfico sexual: el Protocolo de las Naciones Unidas para Reprimir y Sancionar la Trata de Personas, Especialmente Mujeres y Niños (2000) y la Ley de Protección de Víctimas de Tráfico de Estados Unidos (Trafficking Victims Protection Act 2000). Estos dos instrumentos han permitido darle un lugar central al tráfico sexual en las discusiones internacionales sobre la violencia en contra de 
las mujeres y han llevado a que la mayoría de los países adopten regulaciones similares al respecto, en donde se privilegia la criminalización de la conducta y la protección de las personas traficadas (Halley et al., 2018).

En la discusión sobre el alcance, poder y consecuencias de la instalación de las ideas feministas en las instituciones internacionales se ha perdido uno de los puntos centrales de los argumentos de Halley: la relación entre el feminismo de la gobernanza, la estigmatización de la sexualidad y prohibición a través de la criminalización (Franke, 2016). Sin embargo, si se analiza con detenimiento las obras de Halley, se encuentra que una de sus mayores preocupaciones y críticas a la gobernanza internacional es precisamente la tendencia hacia la censura de las conductas sexuales como una herramienta de control social (Halley, 2016, 2008; Halley et al,. 2018).

En este sentido, Lowenkron y Piscitelli (2015) y Vianna y Lowenkron (2017) señalan que las narrativas estatales y transnacionales sobre la comercialización del sexo replican las asimetrías de poder al interior del feminismo, motivo por el cual prevalecen concepciones occidentales y coloniales que se enfocan en la victimización y minimizan la capacidad volitiva de las mujeres. Particularmente, Piscitelli $(2011,2012)$ constata que la conceptualización del trabajo sexual en el debate público difiere de las experiencias de estas mujeres, quienes suelen criticar la estigmatización de su oficio, tienden a temer la deportación más que el riesgo de violencia o explotación y suelen ser excluidas de los centros de toma de decisiones. Bajo esta lógica, las trabajadoras sexuales que defienden la elección de su quehacer son objeto de fuertes críticas por parte de ciertos grupos feministas transnacionales que argumentan que estas experiencias son atípicas y no representativas de la "mayoría" de las personas afectadas por el flagelo de la prostitución (Bernstein, 2007; Piscitelli, 2014). Al censurar las voces de las trabajadoras sexuales, se propagan visiones universalistas $y$ estereotipadas, $y$ generalizaciones que propenden por la formulación de políticas de securitización, victimización y criminalización de la 
cadernos pagu (59), 2020:e205917 El feminismo de la gobernanza en la CEDAW

comercialización del sexo (Vianna y Lowenkron, 2017; Piscitelli, 2014).

La construcción de políticas transnacionales sobre la comercialización del sexo opaca la diversidad y la pluralidad de las experiencias de las mujeres (Lowenkron y Piscitelli, 2015). A partir de la noción de una hermandad femenina, impulsan perspectivas que asemejan la prostitución a la explotación sexual y al tráfico de personas, lo que replica discursos de victimización y protección (Piscitelli, 2014). Para contrarrestar estos procesos de homogeneización, Bernstein insiste que la comercialización del sexo "debe evaluarse en el contexto de las condiciones reales de existencia de las mujeres" (1999, 116 Traducción propia). Así, es necesario examinar las relaciones geopolíticas sobre las que se constituyen las prácticas y discursos regulatorios y analizar la instrumentalización de la comercialización del sexo para reproducir las relaciones de poder y hacer del género una conducta disciplinaria (Vianna y Lowenkron, 2017).

Desde esta perspectiva, los discursos transnacionales sobre la comercialización del sexo adoptan una lógica de la salvación que limita la manera como se puede abordar este fenómeno (AbuLughod, 2002). Como resultado, se evidencia un proceso de homogeneización que sitúa a las mujeres como un grupo único en todos los contextos, lo que replica nociones binarias del sexo/género, invisibiliza las diferencias entre las mujeres e insiste en su opresión y victimización (Mohanty, 1988; Menon, 2015). En suma, las explicaciones sobre la comercialización del sexo privilegian ciertas respuestas institucionales, las cuales reflejan los intereses, agendas $\mathrm{y}$ valores de ciertas vertientes feministas (Narayan, 2006).

En este marco de ideas, esta investigación estudia cómo el Comité de la CEDAW ha planteado las cuestiones relacionadas con la comercialización del sexo a partir de los diferentes enfoques: la criminalización total, la abolición o descriminalización parcial, la descriminalización completa o la legalización. El propósito consiste en evidenciar si en las observaciones finales sobre los informes periódicos de los Estados y en las recomendaciones generales hay 
una tendencia hacia la prohibición y estigmatización de la comercialización del sexo. Este análisis cobra valor porque, como lo establecen Zwingel (2014) y Sosa (2017), el Comité de la CEDAW ha sido uno de las plataformas decisivas para la articulación del feminismo de la gobernanza. A partir de este marco teórico, esta investigación contribuye a la discusión sobre las aproximaciones a la sexualidad en la gobernanza internacional.

\section{Resultados}

\section{El predominio del discurso de la prostitución}

El análisis de los documentos del Comité de la CEDAW revela que en la discusión sobre la comercialización del sexo ha predominado el uso del término "prostitución". De las 37 recomendaciones generales que ha emitido este órgano desde febrero de 1986 hasta marzo de 2018, ninguna utiliza el vocablo "trabajo sexual" mientras que nueve utilizan la palabra "prostitución" (Recomendación General del Comité de la CEDAW Número 19, 24, 26, 30, 32, 33, 34, 35 y 37). Así mismo, de las 425 observaciones finales redactadas desde enero de 2000 hasta marzo de 2018, 328 (77\%) utilizan el vocablo prostitución, con un total de 1759 referencias. Por el contrario, solo 18 (4\%) de las observaciones finales hacen alusión al trabajo sexual con un total de 35 alusiones.

Este examen preliminar evidencia que existe un predominio en el uso del término "prostitución" frente al uso del vocablo "trabajo sexual". Dicha selección de conceptos no es neutra, en la medida en que el empleo de la palabra "prostitución" genera una representación simbólica y legal que estigmatiza la sexualidad y refuerza diversos prejuicios sociales (Adriaenssens et al., 2016; Bindel, 2017; Pheterson, 1990). Por el contrario, la escasa utilización del vocablo trabajo sexual indica que la comercialización del sexo aún no se ha liberado de su carga de valoración moral negativa (Musto y Trajtenberg, 2011; Adriaenssens et al., 2016). 
cadernos pagu (59), 2020:e205917 El feminismo de la gobernanza en la CEDAW

\section{La trata y la explotación de las mujeres que ejercen la prostitución}

El Comité de la CEDAW enmarca la discusión sobre la prostitución en conjunción con la explotación sexual y la trata de personas. Más exactamente, 137 de los 328 documentos que mencionan la prostitución relacionan de alguna manera este fenómeno con la explotación y 106 hacen hincapié en la relación entre la prostitución y la trata de personas. Por ejemplo, el Comité de la CEDAW ha expresado su preocupación con "que las mujeres sigan siendo objeto de explotación sexual en la industria del entretenimiento, en especial con fines de prostitución y producción de películas pornográficas" (CEDAW/C/JPN/CO/7-8/párr. 26b); ha calificado como "insuficiente la información sobre la magnitud del problema de la trata interna y la explotación de la prostitución" (CEDAW/C/PER/CO/7-8/párr. 23); y ha insistido en la promulgación de "leyes para garantizar que se castigue la explotación de las mujeres dedicadas a la prostitución y se endurezcan las penas impuestas a los autores" (CEDAW/C/UGA/CO/7/párr. 28). Adicionalmente, a partir de 2008, la mayoría de estos comentarios aparecen bajo el acápite "Tráfico y explotación de la prostitución", lo que evidencia el enfoque que le tiende a dar el Comité de la CEDAW a este fenómeno. Así mismo, en la Recomendación General N. 19 de 1992, el Comité se remite al artículo 6 de la CEDAW para exigir a los Estados partes que adopten medidas "para suprimir todas las formas de trata de mujeres y explotación de la prostitución de la mujer" (CEDAW/C/GC/19/para.15). En este sentido, el Comité de la CEDAW suele insistir en la necesidad de combatir la trata y la explotación de las mujeres que ejercen la prostitución, siendo estos los nodos más recurrentes en los documentos codificados.

En general, la narrativa del Comité de la CEDAW frente al fenómeno de la prostitución se enfoca particularmente en la relación de este fenómeno con el tráfico y la explotación, lo que puede terminar replicando discursos de victimización y protección. El problema, como resaltan Piscitelli (2014), Bernstein (1999) y Vianna y Lowenkron (2017), es que este discurso produce 
prácticas homogeneizadoras y universales que asemejan la prostitución a la victimización. Bajo esta caracterización, no solo se genera una subjetividad jurídica femenina basada en la falta de agencia, sino que se promueve una criminalización parcial que puede someterlas en muchas circunstancias a situaciones vejatorias y peligrosas.

\section{La reintegración y la rehabilitación}

El Comité de la CEDAW tiende a insistir en la reintegración y rehabilitación de las mujeres que participan en la comercialización del sexo. En 54 de las 328 observaciones finales que mencionan la prostitución, este órgano pide que los Estados desarrollen políticas dirigidas a facilitar la reintegración de las mujeres que ejercen la prostitución y en 47 solicita que los Estados propendan por la rehabilitación de estas mujeres. Por ejemplo, en las observaciones finales de Bosnia y Herzegovina en 2013, Camerún en 2014, Bolivia en 2015 y República Checa en 2016, el Comité de la CEDAW le recomienda a los Estados parte que destinen recursos humanos, técnicos y financieros suficientes para programas de rehabilitación y reintegración para las mujeres que desean abandonar la prostitución, y extender esas medidas a las víctimas de la prostitución forzada. Esta solicitud se repite de forma similar en las observaciones finales de Guinea en 2012, Eritrea en 2015 y Estonia en 2016, entre otras.

En este marco de ideas, el Comité de la CEDAW concibe la rehabilitación como una respuesta adecuada para atender a las mujeres que se ven forzadas a ejercer la prostitución. En línea con sus recomendaciones generales, el Comité de la CEDAW hace un llamado a los Estados parte a desarrollar disposiciones penales, medidas preventivas y programas de rehabilitación que protejan a las mujeres que ejercen la prostitución (CEDAW/C/GC/19/párr.24h). Por ejemplo, en la observación final de Alemania en 2009, este órgano "alienta al Estado parte a que siga formulando estrategias y programas tendientes a evitar que las mujeres se dediquen a la prostitución y a que establezca programas de rehabilitación y 
apoyo para las mujeres y las niñas que desean abandonarla, en particular presentando información sobre opciones de subsistencia y sobre el apoyo a ese respecto" (CEDAW/C/DEU/CO/6/pár. 50). Esta aproximación se reitera en las observaciones finales de Guinea Ecuatorial en 2012, Congo en 2012, Cabo Verde en 2013 y Camerún en 2014, entre otras, en las cuales el Comité de la CEDAW identifica la pobreza y la falta de oportunidades como la causa fundamental de la prostitución. Por consiguiente, este órgano enfatiza las narrativas universales de la victimización que han sido fuertemente criticadas por las feministas postcoloniales (Vianna y Lowenkron, 2017; Piscitelli, 2014). Desde esta óptica, el comercio sexual se entiende primordialmente como una actividad marginal no deseada, motivo por el cual el Estado debe propender por la rehabilitación y reintegración las mujeres que ejercen la prostitución.

\section{Vulnerabilidad y marginalización}

El Comité de la CEDAW suele caracterizar a las mujeres que trabajan en la prostitución como un grupo marginalizado y vulnerable. Por ejemplo, en la Recomendación General N. 24 de febrero de 1999, este órgano cataloga a las mujeres que ejercen la prostitución como "pertenecientes a grupos vulnerables y desfavorecidos como los de las emigrantes, las refugiadas y las desplazadas internas, las niñas y las ancianas, (...) las mujeres autóctonas y las mujeres con discapacidad física o mental" (CEDAW/C/GC/24/párr.6). En particular, sostiene que las mujeres que trabajan en la prostitución son particularmente vulnerables a contraer VIH/SIDA y otras enfermedades de transmisión sexual. Así mismo, según el Comité, "la pobreza y el desempleo también obligan a muchas mujeres, incluso a niñas, a ejercer la prostitución", lo que las torna más vulnerables a la violencia y marginalización (CEDAW/C/GC/19/párr.15).

El Comité de la CEDAW reitera la caracterización unidimensional de la prostitución como una situación de vulnerabilidad y marginalización en la Recomendaciones 
Generales N. 32, 35 y 37, en las cuales insiste que las mujeres que ejercen la prostitución tienen necesidades particulares de protección y asistencia, como también las tienen las mujeres en situación de discapacidad, las niñas no acompañadas fuera de su país de origen, las víctimas de traumas y las víctimas de violencia sexual. Por ejemplo, en la Recomendación General N. 37, establece que se debe brindar atención prioritaria en contextos de desastres naturales producidos por cambio climático "a los grupos de mujeres y niñas más marginados, como los de las minorías indígenas, raciales, étnicas y sexuales, las mujeres y niñas con discapacidad, las adolescentes, las mujeres de edad, las mujeres solteras, las mujeres cabeza de familia, las viudas, las mujeres y las niñas que viven en la pobreza tanto en entornos rurales como urbanos, las mujeres que ejercen la prostitución y las desplazadas internas, las apátridas, las refugiadas, las solicitantes de asilo y las mujeres migrantes" (CEDAW/C/GC/37). Es decir, este órgano incluye la prostitución como uno de los factores que afectan la vida y los derechos de las mujeres.

En este marco de ideas, el Comité de la CEDAW subraya que ciertas situaciones pueden hacer que las mujeres tengan una mayor probabilidad de ser explotadas. Por ejemplo, el Comité reconoce que los conflictos armados conducen a un aumento de la prostitución, motivo por el cual los Estados parte deben adoptar medidas especiales protectoras y punitivas para remediar situaciones de victimización (CEDAW/C/GC/19/para.16). Además, en la Recomendación General No. 30, en la que el Comité analiza la situación de la mujer en contextos de conflicto armado y postconflicto, se reconoce que las mujeres migrantes son más susceptibles a la explotación de la prostitución. Así mismo, en este documento el Comité discute la prostitución forzada en el marco de los conflictos armados. Al relacionar este fenómeno con violencia sexual, el Comité determina que existe un régimen de especial protección enmarcado en el Derecho Internacional Humanitario y reforzado por el Derecho Penal Internacional, el cual permite enmarcar la prostitución forzada como un crimen de guerra, de lesa humanidad o tortura cuando se cumplan los 
cadernos pagu (59), 2020:e205917 El feminismo de la gobernanza en la CEDAW

requisitos legales pertinentes. Por este motivo, el Comité exhorta a los Estados partes a establecer medidas legales punitivas que protejan a las mujeres. Así mismo, en la Recomendación General N. 37 de marzo de 2018, al hacer referencia a la problemática del cambio climático, el Comité identifica a las mujeres en la prostitución no solo como una población marginada y vulnerable, sino a los desastres naturales como factores que incrementan la incidencia de la prostitución forzada. En línea con los hallazgos de otras autoras, la prevalencia de las narrativas sobre marginalización y vulnerabilidad propagan prácticas de securitización que justifican acciones estatales dirigidas a "proteger" y "liberar" a las mujeres del flagelo de la prostitución, lo que impregna la comercialización del sexo en su integridad con una connotación negativa (Vianna y Lowenkron, 2017; Bernstein, 1999).

\section{La criminalización y la victimización}

El Comité de la CEDAW ha manifestado su preocupación por las sanciones penales a las mujeres que ejercen la prostitución, en tanto que considera que esto afianza la explotación sexual y el tráfico de las mujeres (Lituania, 2000). Para este órgano, la criminalización de las víctimas de explotación de la prostitución y el tráfico afecta desproporcionalmente a las mujeres (Croacia, 2015). Por esta razón, en las observaciones sobre Burundi 2001, Bélgica 2002, Filipinas 2006, Indonesia 2007, Federación Rusa 2010 y Bielorrusia 2016, entre otras, ha recomendado a los Estados que sancionen a todos los que explotan a las mujeres que ejercen la prostitución. En este sentido, el Comité ha sostenido a través de los años una posición que ha propendido por la no criminalización de las mujeres que se dedican a la prostitución (Ver las observaciones finales de Guinea 2001, Guyana 2001, Albania 2003, China 2006, Corea 2007, Siria 2007, Burundi 2008, Ruanda 2009, Fiyi 2010, Kenia, 2011, Bahamas 2012, Camerún 2014, Ghana 2014, Canadá 2016). Sin embargo, el Comité de la CEDAW ha abogado de manera constante por la criminalización 
parcial del tráfico de personas con fines de prostitución y la explotación de la prostitución. Así, en ciertas observaciones finales, el Comité ha propendido por la criminalización específica del proxenetismo, tales como en el caso de Saint Kitts y Nevis en 2002, Ecuador en 2003 y China en 2006.

El análisis de estos documentos permite identificar en la narrativa del Comité la preponderancia de un enfoque abolicionista que privilegia el derecho penal como estrategia primordial para lograr la erradicación de la prostitución. Esto implica adoptar una lectura de la comercialización del sexo como una relación de dominación de hombres hacia las mujeres donde no existen matices (Bernstein, 1999). De esta manera, el derecho internacional se transforma en un instrumento de gobernanza que exige de los Estados parte la adopción de medidas de criminalización que pueden poner en mayor peligro a las mujeres que se dedican al comercio sexual (Lowenkron y Piscitelli, 2015; Piscitelli, 2014). Penalizar de manera indiscriminada a cualquiera de las personas que participan en el comercio sexual generalmente obliga a las mujeres que derivan de este su sustento a trabajar en condiciones de riesgo, tales como lugares desolados, y a desarrollar relaciones de desconfianza con las autoridades (Adriaenssens et al., 2016; Bernstein, 2007). Bajo este marco lógico, las mujeres son las víctimas en la comercialización del sexo y el Estado debe adoptar medidas punitivas para prevenir este flagelo, lo que implica perseguir a todos aquellos involucrados en este tipo de actividades.

\section{El problema de la demanda}

En 33 documentos, el Comité de la CEDAW recomienda que los Estados adopten políticas encaminadas a reducir la demanda de la prostitución. En este sentido, el Comité ha condenado el que no se criminalice la demanda de servicios sexuales. Así ocurrió en las observaciones finales de Egipto en el año 2001, Mauritius en el 2006 y Ghana en 2014. También, ha recomendado específicamente la criminalización de la demanda servicios 
cadernos pagu (59), 2020:e205917 El feminismo de la gobernanza en la CEDAW

sexuales, como en el caso de las observaciones finales para Bélgica y Finlandia en el 2014; y ha felicitado a países como Suecia en 2001 por haber introducido esta medida. A pesar de que la recomendación de la criminalización de la demanda parece ser una línea clara en la aproximación del Comité de la CEDAW, este ha hecho algunas veces llamados para que se evalúen las posibles consecuencias negativas de esta medida. Por esto, en el caso referido de Suecia, la observación final indicó que era necesario que este país evaluara si la criminalización de la demanda había incrementado la prostitución clandestina, con todo lo que ello implica para la integridad de las mujeres dedicadas al comercio sexual. Para el caso de Noruega, la observación final de 2007 hizo un llamado para que llevara a cabo un estudio profundo de las posibles consecuencias de su plan de implementar esta medida. Igualmente, en la observación final de Francia en el 2016, este órgano señaló que esta podía no tener los efectos deseados, por cuanto no solo ponía en peligro la seguridad y salud de las mujeres trabajando en prostitución, sino que no atacaba las causas de esta o disminuía su incidencia. A pesar de la preferencia del Comité de la CEDAW por la criminalización de la demanda, este también ha recomendado tomar medidas educativas para el efecto. Es el caso, por ejemplo, de las observaciones finales de Bielorrusia en 2016 y Fiyi en 2018.

En general, el Comité de la CEDAW reitera que los Estados deben condenar la demanda de servicios sexuales. Así, es posible concluir que el Comité se inclina hacia la abolición o descriminalización parcial de la prostitución, la cual no penaliza a las trabajadoras sexuales, pero que sí busca sancionar a los actores involucrados en la industria sexual, incluyendo a los consumidores. De acuerdo con estos hallazgos, se encuentra que el Comité concibe la prostitución como una manifestación de la explotación y tráfico sexual de la mujer. Por consiguiente, no hay cabida para el consentimiento, en tanto que la prostitución es vista como una de las expresiones de dominación y violencia a las que están sometidas las mujeres. Esta aproximación no solo puede someter a las mujeres que trabajan en el comercio sexual a 
situaciones de mayor desprotección, sino consolida una idea de ser mujer anclada en la victimización y la falta de autonomía sobre sus cuerpos (Lowenkron y Piscitelli, 2015; Vianna y Lowenkron, 2017).

\section{Trabajo sexual}

Como se mencionó anteriormente, de las 37 recomendaciones generales que ha emitido este órgano desde febrero de 1986 hasta marzo de 2018, ninguna utiliza el vocablo "trabajo sexual". Así mismo, de las 425 observaciones finales redactadas desde enero de 2000 hasta marzo de 2018, solo 18 (4\%) hacen alusión al trabajo sexual con un total de 35 alusiones. La mayoría de estas referencias aparecen cuando el Comité de la CEDAW estudia las situaciones de Estados en donde el comercio sexual está legalizado, como en el caso de Bolivia y Alemania, o para Estados que no criminalizan a la mujer que lo ejerce, como en el caso de Belice, Estonia y Noruega, entre otros. Sin embargo, también este concepto es utilizado en observaciones finales respecto de Estados que prohíben la comercialización del sexo, como es el caso de Ghana, Gambia, Camerún, Azerbaiyán y Siria, pero en los que es una práctica común (UNAIDS, 2016).

En ocho de las 18 referencias sobre trabajo sexual, el Comité de la CEDAW manifiesta su preocupación por la salud de las mujeres que comercializan su cuerpo. Además, en cuatro documentos, insiste en la importancia de brindarle a las trabajadoras sexuales opciones para que puedan ejercer otra profesión. En este sentido, el uso del vocablo trabajo sexual no modifica drásticamente la forma en como este órgano problematiza la comercialización del sexo. Es decir, el trabajo sexual continúa siendo una práctica que afecta los derechos humanos y la salud de las mujeres. Así, el uso de la expresión "trabajo sexual" no indica el surgimiento de una aproximación diferente a la comercialización del sexo por parte del Comité de la CEDAW.

En los documentos analizados, no fue posible encontrar aproximaciones a la comercialización del sexo en las que se 
cadernos pagu (59), 2020:e205917 El feminismo de la gobernanza en la CEDAW

examinaran de manera explícita la posibilidad de que esta actividad fuera ejercida de manera voluntaria $y$ en contextos ajenos a los de explotación. Si bien no hay un llamado concreto a la eliminación de la prostitución, la insistencia del Comité de la CEDAW en la dimensión de victimización de las mujeres en el comercio sexual evidencia que en este órgano prevalece una aproximación feminista de criminalización que entrelaza tráfico, prostitución y explotación de la mujer.

\section{Conclusión}

Esta investigación analizó cómo el Comité de la CEDAW aborda la comercialización del sexo. Para ello, partió del concepto del feminismo de la gobernanza acuñado por Halley et al. (2006), para rastrear qué ideas feministas han logrado convertirse en parte de los dispositivos de regulación de las conductas humanas a nivel de derecho internacional en este tema. El objetivo era comprobar a través del análisis cualitativo de las recomendaciones generales y observaciones finales del Comité de la CEDAW si efectivamente las ideas feministas radicales habían logrado insertarse en la narrativa de este órgano.

Al contrastar los resultados con la posición de Halley et al. (2006) se encuentra que efectivamente el Comité de la CEDAW ha promovido primordialmente un enfoque de descriminalización parcial o abolicionista que pretende penalizar a aquellos que participen en el comercio sexual, salvo la mujer en prostitución, la cual es considerada primordialmente víctima. En términos generales, el Comité caracteriza la prostitución como una situación de vulnerabilidad y marginalización e insiste en la no discriminación e igualdad de las mujeres que ejercen la prostitución. Así, el Comité tiende a centrarse en la explotación de la prostitución y el tráfico. En este sentido, el Comité parece caracterizar la prostitución exclusivamente como un fenómeno de explotación, violencia y tráfico.

La compaginación de la prostitución con el tráfico y la explotación sexual, y el silencio ante la posibilidad de medidas 
para la regulación del trabajo sexual reafirman que el Comité se aproxima a este tema desde una perspectiva primordialmente abolicionista. Por esta razón, la comercialización del sexo como un todo permanece atada a su carga de valoración negativa y universalista que tiende a anclar versiones y construcciones jurídicas que victimizan a todas las mujeres que participan en esta. Cabe preguntarse si esta representación simbólica y legal refuerza diversos perjuicios sociales que estigmatizan la sexualidad de la mujer $e$ impide avanzar en respuestas institucionales que contemplen las situaciones en donde prima la voluntariedad de la comercialización del sexo.

\section{Referências bibliográficas}

ABU-LUGHOD, Lila. "Do Muslim Women Really Need Saving? Anthropological Reflections on Cultural Relativism and Its Others." American Anthropologist $104 \quad$ (3), 2002, pp.783-90 [www.jstor.org/stable/3567256 - accesado el 7 Octubre 2020].

ADRIAENSSENS, Stef, Giulia Garofalo Geymonat, Laura Oso, K U Leuven, and Martin A Monto. "Female Prostitution, Customers, and Violence." Sociological Research Online 10 (2), 2016, pp.1-12 [https://doi.org/10.5153/sro.4165 - accesado el 7 Octubre 2020].

BERNSTEIN, Elizabeth. "What's Wrong with Prostitution? What's Right with Sex Work? Comparing Markets in Female Sexual Labor." Hastings Women's Law Journal 10 (1), 1999, pp.91-117 [Accesado el 7 Octubre 2020].

. "Sex Work for the Middle Classes." Sexualities 10 (4), 2007. pp.473-88 [https://doi.org/10.1177/1363460707080984 - accesado el 7 Octubre 2020].

BINDEL, Julie. The Pimping of Prostitution: Abolishing the Sex Work Myth. Londres: Springer, 2017.

COMITÉ PARA LA ELIMINACIÓN DE LA DISCRIMINACIÓN CONTRA LA MUJER (CEDAW), Recomendación General No 19: La violencia contra la Mujer. CEDAW, 29 enero 1992 [https://www.refworld.org.es/docid/5d7fbd535.html - accesado el 3 Septiembre 2020]. 
cadernos pagu (59), 2020:e205917 El feminismo de la gobernanza en la CEDAW

. Recomendación General No 24: La mujer y la salud. CEDAW, 2 febrero 1999 [https://www.refworld.org.es/docid/5d7fbd5113.html accesado el 3 Septiembre 2020].

. Recomendación General No 37: Dimensiones de género de la reducción del riesgo de desastres en el contexto del cambio climático. CEDAW, 13 marzo 2018 [https:/undocs.org/CEDAW/C/GC/37 accesado el 3 Septiembre 2020].

. Observaciones finales sobre los informes periódicos Alemania, 12 de febrero de 2009, CEDAW/C/DEU/CO/6 [https://undocs.org/es/CEDAW/C/DEU/CO/6 - accesado el 3 Septiembre 2020].

. Observaciones finales sobre los informes periódicos Uganda, 3 de noviembre de 2010, CEDAW/C/UGA/CO/7 [https://tbinternet.ohchr.org/_layouts/15/treatybodyexternal/Download .aspx?symbolno $=\mathrm{CEDAW} / \overline{\mathrm{C}} / \mathrm{UGA} / \mathrm{CO} / 7 \& \mathrm{Lang}=\mathrm{Sp}-$ accesado el 3 Septiembre 2020].

. Observaciones finales sobre los informes periódicos séptimo y octavo combinados del Perú, 24 de julio de 2014, $\mathrm{CEDAW} / \mathrm{C} / \mathrm{PER} / \mathrm{CO} / 7$

[https://tbinternet.ohchr.org/_layouts/15/treatybodyexternal/Download .aspx?symbolno $=\mathrm{CEDAW} / \overline{\mathrm{C}} / \mathrm{PER} / \mathrm{CO} / 7-8 \& \mathrm{Lang}=\mathrm{Sp}-$ accesado el 3 Septiembre 2020].

. Observaciones finales sobre los informes periódicos séptimo y octavo combinados del Japón, 10 marzo 2016, $\mathrm{CEDAW} / \mathrm{C} / \mathrm{JPN} / \mathrm{CO} / 7-8$

[https://tbinternet.ohchr.org/ layouts/15/treatybodyexternal/Download aspx? symbolno $=\mathrm{CEDAW} / \mathrm{C} / \mathrm{JPN} / \mathrm{CO} / 7-8 \& \mathrm{Lang}=\mathrm{En}$ - accesado el 3 Septiembre 2020].

CÉSPEDES-BÁEZ, Lina M. Far beyond What Is Measured: Governance Feminism and Indicators in Colombia. International Law, n. 25, 2014, pp.375-444

[http://www.scielo.org.co/scielo.php?script=sci abstract\&pid $=S 1692$ $\underline{81562014000200008}$ - accesado el 7 Octubre 2020].

CHARLESWORTH, Hilary. "Are Women Peaceful? " Reflections on the Role of Women in Peace-Building. Feminist Legal Studies 16 (3), 
2008, pp.347-61 [https://doi.org/10.1007/s10691-008-9101-6 accesado el 7 Octubre 2020].

. Talking to Ourselves: Should International Lawyers Take a Break from Feminism. In: KOUVO, Sari; PEARSON, Zoe (ed.). Feminist Perspectives on Contemporary International Law: Between Resistance, Hart Publishing, 2011, pp.17-32.

CHOUDHURY, Cyra Akila. Globalizing the Margins: Legal Exiles in the War on Terror and Liberal Feminism's War for Muslim Women. International Review of Constitutionalism 9 (2), 2010, pp.241-61.

CRENSHAW, Kimberle. Mapping the Margins: Intersectionality, Identity Politics, and Violence against Women of Color. Stanford Law Review 43 (6), 1991, pp.1241-99 [https://doi.org/10.2307/1229039 accesado el 7 Octubre 2020].

DIETZ, Mary G. Current Controversies in Feminist Theory. Annual Review of Political Science 6 (1), 2003, pp.399-431 [https://doi.org/10.1146/annurev.polisci.6.121901.085635 - accesado el 7 Octubre 2020].

FRANKE, Katherine M. Theorizing Yes: An Essay on Feminism, Law, and Desire. In: Feminist and Queer Legal Theory, Routledge. 2016. pp.43-58 [www.jstor.org/stable/1123512 - accesado el 7 Octubre 2020].

HALLEY, Janet. Split Decisions: How and Why to Take a Break from Feminism. Princeton University Press, 2008.

. The Move to Affirmative Consent. Signs: Journal of Women in Culture and Society $42 \quad$ (1), $2016 \quad$ pp.257-79 [https://doi.org/10.1086/686904 - accesado el 7 Octubre 2020].

HALLEY, Janet et al. Introducing Governance Feminism. In Governance Feminism: An Introduction, ix-xxi. Minneapolis, University of Minnesota Press, 2018.

HALLEY, Janet et al. From the International to the Local in Feminist Legal Responses to Rape, Prostitution/Sex Work, and Sex Trafficking: Four Studies in Contemporary Governance Feminism. Harv. $J L \&$ Gender 29, 2006, pp.335-423 [https://ssrn.com/abstract=1438140 accesado el 7 Octubre 2020]. 
cadernos pagu (59), 2020:e205917 El feminismo de la gobernanza en la CEDAW

JARAMILlO RUIZ, Felipe. Democracy, Parity and Change. An Institutional Space for Gender Relations. Civilizar 13 (25), 2013, pp.67-84 [http://www.redalyc.org/articulo.oa?id=100230029004 accesado el 7 Octubre 2020].

JARAMILLO RUIZ, Felipe; ERAZO, Catalina. Gender equality as a general interest of a pluralistic society: an analysis of constitutional precedent in Colombia. Bulletin of Latin American Research 35 (2), 2016, pp.178-90 [https://doi.org/10.1111/blar.12370 - accesado el 7 Octubre 2020].

LOWENKRON, Laura; PISCITELLI, Adriana. Trabajadoras sexuales, policía, migración y trata internacional de seres humanos enlos dos lados del océano. In: DAICH, Deborah; SIRIMARCO, Mariana (ed.). Género y violencia en el mercado del sexo: política, policía y prostitución. Buenos Aires, Biblos., 2015, pp.173-203.

MACKINNON, Catharine A. Feminism, Marxism, Method, and the State: Toward Feminist Jurisprudence. Signs 8 (4), 1983, pp.635-58 [http://www.jstor.org/stable/3173687 - accesado el 7 Octubre 2020].

MENON, Nivedita. Is Feminism about 'Women'? Economic and Political Weekly, 2015

[https://www.epw.in/journal/2015/17/perspectives/feminism-aboutwomen.html - accesado el 7 Octubre 2020].

MILLER, Coty R.; HALTIWANGER, Nuria. Prostitution and the Legalization/Decriminalization Debate. Geo. J. Gender \& L. 5, 2004, pp.207-35

[https://heinonline.org/HOL/LandingPage?handle=hein.journals/grgg enl5\&div =18\&id=\&page $=$ - accesado el 7 Octubre 2020] .

MOHANTY, Chandra Talpade. Under Western Eyes: Feminist Scholarship and Colonial Discourses. Feminist Review, no. 30, 1988, pp.61-88 [doi:10.2307/302821 - accesado el 7 Octubre 2020].

MONROY, María Catalina; JARAMILLO RUIZ, Felipe. Violencia urbana: la red mujer y hábitat de América Latina (el caso de Colombia). Revista CIDOB d'Afers Internacionals, n. 117, 2017, pp.123-43 [doi.org/10.24241/rcai.2017.117.3.123 - accesado el 7 Octubre 2020].

MUSTO, Clara; TRAJTENBERG, Nico. Prostitución y trabajo sexual: el estado de arte de la investigación en Uruguay. Revista de Ciencias 
Sociales, n. 29, 2011, pp.139-56 [ttps://www.redalyc.org/articulo.oa?id $=453644790008$ - accesado el 7 Octubre 2020].

NARAYAN, Uma. Cross-Cultural Connections, Border-Crossings, and 'Death by Culture': Thinking about Dowry-Murders in India and Domestic-Violence Murders in the United States. Dislocating Cultures: Identities, Traditions, and Third World Feminism, 2006, pp.83-117.

OTTO, Dianne. International Human Rights Law: Towards Rethinking Sex/Gender Dualism and Asymmetry (November 20, 2012). Forthcoming in A Research Companion to Feminist Legal Theory, M. Davies and V. Munro. Ashgate, 2010a.

. Power and Danger: Feminist Engagement with International Law through the UN Security Council. Australian Feminist Law Journal 32 (1), $2010 \mathrm{~b}$ pp.97-121 [https://doi.org/10.1080/13200968.2010.10854439 - accesado el 7 Octubre 2020].

PHETERSON, Gail. The Category 'Prostitute' in Scientific Inquiry. The Journal of Sex Research 27 (3), 1990, pp.397-407 [https://doi.org/10.1080/00224499009551568 - accesado el 7 Octubre 2020].

PISCITELLI, Adriana. El debate feminista y la discusión sobre prostitución, reflexiones a partir de Brasil. In La Antropología Feminista Hoy: Desafíos Teóricos y Políticos En Un Mundo Globalizado. Argentina, 2011.

Revisiting Notions of Sex Trafficking and Victims. Vibrant: Virtual Brazilian Anthropology 9 (1), pp.274-310 [http://www.scielo.br/scielo.php?script=sci_arttext\&pid=S180943412012000100010\&nrm =iso - accesado el 7 Octubre 2020].

. Transnational Sisterhood? Brazilian Feminisms Facing Prostitution. Latin American Policy 5 (2), 2014, pp.221-35 [https://doi.org/10.1111/lamp.12046 - accesado el 7 Octubre 2020].

PRÜGL, Elisabeth. Diversity Management and Gender Mainstreaming as Technologies of Government. Politics \& Gender 7 (1), 2011, pp.7189. 
cadernos pagu (59), 2020:e205917 El feminismo de la gobernanza en la CEDAW

. Neoliberalism with a Feminist Face: Crafting a New Hegemony at the World Bank. Feminist Economics 23 (1), 2017, pp.30-53 [https://doi.org/10.1080/13545701.2016.1198043 - accesado el 7 Octubre 2020].

SOSA, Lorena. Intersectionality in the Human Rights Legal Framework on Violence against Women: At the Centre or the Margins? Cambridge University Press, 2017.

STABILE, Carol A,; KUMAR, Deepa. Unveiling Imperialism: Media, Gender and the War on Afghanistan. Media, Culture \& Society 27 (5), 2005 pp.765-82 [https://doi.org/10.1177\%2F0163443705055734 - accesado el 7 Octubre 2020].

VERSCHUUR, Christine. A History of Development Through a Gender Prism: Feminist and Decolonial Perspectives. In: VERSCHUUR, Christine; GUÉRIN, Isabelle; GUÉTAT-BERNARD, Hélène (ed.). Under Development: Gender. London, Palgrave Macmillan UK, 2014, pp.17-41 [https://doi.org/10.1057/9781137356826_2 - accesado el 7 Octubre 2020].

VIANNA, Adriana; LOWENKRON, Laura. O duplo fazer do gênero e do estado: interconexões, materialidades e linguagens. cadernos pagu, $\mathrm{n}$. 51 , 2017

[http://www.scielo.br/scielo.php?script=sci_arttext\&pid=S010483332017000300302\&nrm =iso - accesado el 7 Octubre 2020].

ZWINGEL, Sussanne. Translating International Women's Rights, The CEDAW Convention in Context. Palgrave, 2014. 